\title{
Association of Serum Soluble Urokinase Receptor Levels With Progression of Kidney Disease in Children
}

\author{
Franz Schaefer, MD; Howard Trachtman, MD; Elke Wühl, MD; Marietta Kirchner, PhD; Salim S. Hayek, MD; Ali Anarat, MD; Ali Duzova, MD; \\ Sevgi Mir, MD; Dusan Paripovic, MD; Alev Yilmaz, MD; Francesca Lugani, MD; Klaus Arbeiter, MD; Mieczyslaw Litwin, MD; Jun Oh, MD; \\ Maria Chiara Matteucci, MD; Jutta Gellermann, MD; Simone Wygoda, MD; Augustina Jankauskiene, MD; Günter Klaus, MD; Jiri Dusek, MD; \\ Sara Testa, MD; Aleksandra Zurowska, MD; Alberto Caldas Afonso, MD; Melissa Tracy, MD; Changli Wei, MD, PhD; Sanja Sever, PhD; \\ William Smoyer, MD; Jochen Reiser, MD, PhD; for the ESCAPE Trial Consortium and the 4C Study Group
}

IMPORTANCE Conventional methods to diagnose and monitor chronic kidney disease (CKD) in children, such as creatinine level and cystatin C-derived estimated glomerular filtration rate (eGFR) and assessment of proteinuria in spot or timed urine samples, are of limited value in identifying patients at risk of progressive kidney function loss. Serum soluble urokinase receptor (suPAR) levels strongly predict incident CKD stage 3 in adults.

OBJECTIVE To determine whether elevated suPAR levels are associated with renal disease progression in children with CKD.

DESIGN, SETTING, AND PARTICIPANTS Post hoc analysis of 2 prospectively followed up pediatric CKD cohorts, ie, the ESCAPE Trial (1999-2007) and the 4C Study (2010-2016), with serum suPAR level measured at enrollment and longitudinal eGFR measured prospectively. In the 2 trials, a total of 898 children were observed at 30 (ESCAPE Trial; $\mathrm{n}=256$ ) and 55 (4C Study; $n=642$ ) tertiary care hospitals in 13 European countries. Renal diagnoses included congenital anomalies of the kidneys and urinary tract $(n=637$ [70.9\%]), tubulointerstitial nephropathies ( $n=92$ [10.2\%]), glomerulopathies ( $n=69$ [7.7\%]), postischemic CKD ( $n=42[4.7 \%])$, and other CKD ( $n=58$ [6.5\%]). Total follow-up duration was up to 7.9 years, and median follow-up was 3.1 years. Analyses were conducted from October 2016 to December 2016.

EXPOSURES Serum SUPAR level was measured at enrollment, and eGFR was measured every 2 months in the ESCAPE Trial and every 6 months in the 4C Study. The primary end point of CKD progression was a composite of $50 \%$ eGFR loss, eGFR less than $10 \mathrm{~mL} / \mathrm{min} / 1.73 \mathrm{~m}^{2}$, or initiation of renal replacement therapy.

MAIN OUTCOMES AND MEASURES The primary end point in this study was renal survival, defined as a composite of $50 \%$ loss of GFR that persisted for at least 1 month, the start of renal replacement therapy, or an eGFR less than $10 \mathrm{~mL} / \mathrm{min} / 1.73 \mathrm{~m}^{2}$.

RESULTS Of the 898 included children, 560 (62.4\%) were male, and the mean (SD) patient age at enrollment was 11.9 (3.5) years. The mean (SD) eGFR was $34(16) \mathrm{mL} / \mathrm{min} / 1.73 \mathrm{~m}^{2}$. The 5 -year end point-free renal survival was $64.5 \%(95 \% \mathrm{Cl}, 57.4-71.7)$ in children with suPAR levels in the lowest quartile compared with $35.9 \%(95 \% \mathrm{Cl}, 28.7-43.0)$ in those in the highest quartile $(P<.001)$. By multivariable analysis, the risk of attaining the end point was higher in children with glomerulopathies and increased with age, blood pressure, proteinuria, and lower eGFR at baseline. In patients with baseline eGFR greater than $40 \mathrm{~mL} / \mathrm{min} / 1.73 \mathrm{~m}^{2}$, higher log-transformed suPAR levels were associated with a higher risk of CKD progression after adjustment for traditional risk factors (hazard ratio, 5.12; $95 \% \mathrm{Cl}, 1.56-16.7 ; P=.007$ ).

CONCLUSIONS AND RELEVANCE Patients with high suPAR levels were more likely to have progression of their kidney disease. Further studies should determine whether suPAR levels can identify children at risk for future CKD.

JAMA Pediatr. 2017;171(11):e172914. doi:10.1001/jamapediatrics.2017.2914

Published online September 5, 2017. Corrected on October 9, 2017.
Supplemental content

Author Affiliations: Author affiliations are listed at the end of this article.

Group Information: Members of the ESCAPE Trial Consortium and the $4 C$ Study Group are listed at the end of this article.

Corresponding Author: Jochen Reiser, MD, PhD, Department of Medicine, Rush University Medical Center, Kellogg Bldg, Ste 1003 , Chicago, IL 60612 (jochen_reiser@rush.edu). 
C hronic kidney disease (CKD) in children is associated with significant comorbidities, compromising life expectancy, quality of life, and psychosocial integration during childhood and early adult life. ${ }^{1,2}$ Although pediatric CKD is most commonly caused by congenital renal malformations and hereditary disorders, stringent blood pressure control and antiproteinuric pharmacotherapy have been established in the ESCAPE Trial (Effect of Strict Blood Pressure Control and ACE Inhibition on the Progression of CRF in Pediatric Patients; NCT00221845) ${ }^{3}$ as the first effective renoprotective strategy in pediatric CKD. Post hoc analyses and observational studies have identified additional modifiable conditions that effect the rate of deterioration in kidney function, such as metabolic acidosis and vitamin D deficiency. ${ }^{4,5}$ A growing body of preclinical and clinical research is evaluating novel approaches to pharmacological nephroprotection that may become available for children with CKD in the foreseeable future. ${ }^{6,7}$

The individual rate of eGFR loss in children is highly variable, in part because of the heterogeneity of underlying renal diseases and the differential effect of age and possibly sex. However, progression rates vary widely even within individual age and disease groups, indicating a need for informative prognostic biomarkers. Estimation of the glomerular filtration rate from serum creatinine level and determination of proteinuria have traditionally been used to identify patients at heightened risk of developing progressive CKD. ${ }^{1,2}$ Although a variety of novel urinary biomarkers of tubular injury, including neutrophil gelatinase-associated lipocalin, kidney injury molecule-1, and renal liver-type fatty acid binding protein, have been proposed to improve the accuracy of predicting the development of CKD, they have not been found to improve predictive models of CKD based on traditional laboratory tests in adults. ${ }^{8,9}$

Recently, soluble urokinase receptor (suPAR), a marker of immune activation involved in the pathogenesis of focal segmental glomerulosclerosis, ${ }^{10}$ was found to be strongly predictive of CKD beyond traditional clinical measures in adult patients. ${ }^{11}$ These findings have subsequently been confirmed in 2 other adult cohorts. ${ }^{12,13}$ Whether suPAR levels are associated with decline in renal function in the pediatric CKD population is unknown. In this report, we present a post hoc analysis of a combined cohort consisting of pediatric patients with CKD enrolled in 2 studies: the observational 4C Study (Cardiovascular Comorbidity in Children with Chronic Kidney Disease Study; NCTO1046448) $)^{14,15}$ and the ESCAPE Trial. ${ }^{3}$ We tested the hypothesis that high serum suPAR levels may be a biomarker in children with CKD that is associated with a more rapid decline in renal function.

\section{Methods}

This study follows the Strengthening the Reporting of Observational Studies in Epidemiology (STROBE) Statement checklist for cohort studies (https://www.strobe-statement.org). ${ }^{16}$

\section{Study Population}

The effect of suPAR serum levels on eGFR decline in children with CKD was analyzed by combining 2 of the largest pediatric

\section{Key Points}

Question Are elevated serum soluble urokinase receptor (suPAR) levels associated with renal disease progression in children with chronic kidney disease?

Findings In this cohort study of 898 children, 5-year end point-free renal survival was $64.5 \%$ in children with suPAR levels in the lowest quartile compared with $35.9 \%$ in those with suPAR levels in the highest quartile. In patients with baseline estimated glomerular filtration rates greater than $40 \mathrm{~mL} / \mathrm{min} / 1.73 \mathrm{~m}^{2}$, higher log-transformed suPAR levels were associated with a higher risk of chronic kidney disease progression after adjusting for conventional risk factors.

Meaning High suPAR levels may identify children at risk for future chronic kidney disease.

CKD cohorts: the 4C Study ${ }^{14,15}$ and the ESCAPE Trial. ${ }^{3}$ Both studies were designed and performed according to the Declaration of Helsinki. The protocols were approved by the central ethics committee of the Heidelberg University Medical Faculty and by each local ethical committee. Written informed consent, including future use of stored specimens, was obtained from the parents and adolescents, and oral informed assent was obtained from younger children. Patients were eligible for inclusion in this study if (1) they were participants in the 4C Study or ESCAPE Trial, (2) data on renal disease progression during follow-up were available, and (3) serum samples were available for determination of suPAR concentration.

In the 4C Study, 704 patients were enrolled to study the causes and consequences of cardiovascular disease in children and adolescents aged 6 to 17 years with CKD stage 3B or 4 (eGFR, $15-45 \mathrm{~mL} / \mathrm{min} / 1.73 \mathrm{~m}^{2}$ ) prior to dialysis or transplantation. The children were observed prospectively, with clinical assessments every 6 months and collection of blood and urine samples. ${ }^{14,15}$

The ESCAPE Trial was an investigator-initiated randomized clinical trial evaluating the effect of intensified blood pressure control on renal disease progression. A total of 468 children aged 3 to 17 years with CKD stage 2 to 4 (GFR, 15 to $80 \mathrm{~mL} / \mathrm{min} / 1.73 \mathrm{~m}^{2}$ ) were enrolled. All patients received the angiotensin-converting enzyme inhibitor ramipril in a fixed dose of $6 \mathrm{mg} / \mathrm{m}^{2} / \mathrm{d}$ and additional antihypertensive medication, where appropriate, targeting a blood pressure goal of either below the 50th or between the 50th and 95th blood pressure percentile. Children were followed up prospectively, with clinical assessments every 2 months and blood and urine collection. ${ }^{3}$

\section{Laboratory Assessment}

In both studies, urinary analyses and renal function parameters were analyzed centrally, using standard laboratory techniques to measure the serum and urinary concentrations of creatinine and urinary protein excretion, as described previously. ${ }^{3,14,15}$ In the 4C Study, eGFR was determined using cystatin $\mathrm{C}$ clearance and proteinuria was measured as the albumin:creatinine ratio in spot urines. In the ESCAPE Trial, eGFR was calculated using the Schwartz formula, and proteinuria was measured as the protein:creatinine ratio in a 24-hour urine 
sample. Serum suPAR levels were measured with a commercially available enzyme-linked immunosorbent assay kit (suPARnostic kit; ViroGates), as described previously. ${ }^{11}$ The intra-assay and interassay variability for the suPAR determinations were $2.75 \%$ and $9.17 \%$, respectively. We have demonstrated that serum suPAR levels are stable and reproducible in samples stored for more than 5 years at $-70^{\circ} \mathrm{C} .{ }^{11}$

\section{Statistical Analysis}

Baseline characteristics of the whole sample and stratified by study (4C vs ESCAPE) are described by means and SDs, medians and interquartile ranges, and frequencies. Proteinuria was defined as positive or negative by either urinary protein excretion of $0.5 \mathrm{~g} / \mathrm{g}$ creatinine or greater (ESCAPE Trial) or albumin excretion of $50 \mathrm{mg} / \mathrm{g}$ creatinine or greater (4C Study). The influence of sex, diagnoses, initial eGFR, and age on logtransformed suPAR levels was analyzed by a multivariable linear model. In addition, the association was adjusted for study (ESCAPE Trial vs 4C Study).

The primary end point in this study was renal survival, defined as a composite of $50 \%$ loss of GFR that persisted for at least 1 month, the start of renal replacement therapy, or an eGFR less than $10 \mathrm{~mL} / \mathrm{min} / 1.73 \mathrm{~m}^{2}$. If a $50 \%$ reduction in eGFR occurred between 2 study visits, interpolation was used to determine the time of the event. Kaplan-Meier curves stratified by quartiles of the suPAR levels are presented. Cox proportional regression models were applied to evaluate associations between baseline covariate levels and time to composite event. Three models were considered, which all included sex, age, proteinuria, systolic blood pressure, and renal diagnoses as covariates as well as study as a strata variable. In model 1, eGFR was added to the set of 5 basic covariates; in model 2, the log-transformed baseline suPAR level was added; and in model 3, both eGFR and the log-transformed baseline suPAR level were added to the basic covariates. Quality of the models was compared based on the Akaike information criterion (AIC), with lower values indicating a better fit. The AIC value is the expected, relative distance between the fitted model and the unknown true mechanism that generated the observed data. Model fits were compared by transformation of AIC to Akaike weights. ${ }^{17}$ Akaike weights can be interpreted as the probability that the respective model is the best model.

Additionally, hazard ratios for renal survival for high suPAR levels (ie, greater than the median) vs low suPAR levels were analyzed. Covariates included in the model were baseline eGFR, age, systolic blood pressure, standard deviation score, sex, proteinuria (categorized as proteinuria yes/ no), and diagnosis. In every model, the interaction of suPAR with the respective subgroup factor was included, and the $\chi^{2}$ $P$ value is reported. All $P$ values are 2-tailed, and significance was set at $P<.05$. Furthermore, sensitivity analyses using different eGFR cutoff values were performed to determine whether the association between suPAR level and the primary end point varied according to baseline eGFR. Based on previous studies, ${ }^{11,18}$ we used an eGFR cutoff of $40 \mathrm{~mL} / \mathrm{min} /$ $1.73 \mathrm{~m}^{2}$ to examine the differential association between suPAR level and eGFR decline in patients with and without severe renal dysfunction.

\section{Results}

\section{Cohort Description}

A total of 898 children (642 [71.5\%] from the 4C Study and 256 [28.5\%] from the ESCAPE Trial) with stored serum available for assay of serum suPAR concentration at baseline were included in the present analysis. The mean (SD) age of the combined study population was 11.9 (3.5) years, and the mean (SD) eGFR was 34 (16) $\mathrm{mL} / \mathrm{min} / 1.73 \mathrm{~m}^{2}$ (Table 1). The median (interquartile range) duration of follow-up was 3.1 (1.3-7.5) years, with a range of 0 to 7.9 years. The primary renal diagnoses were categorized as glomerulopathies, uropathic congenital anomalies of the kidneys and urinary tract (CAKUT; refluxive/ obstructive CAKUT), other CAKUT (ie, renal hypoplasia or dysplasia without uropathy), reduced renal mass subsequent to acute kidney injury, tubulointerstitial nephropathies (including metabolic kidney disorders), and other or unknown kidney diseases. The CAKUT categories combined accounted for 637 of 898 children (70.9\%) in the cohort, and only $69(7.7 \%)$ had a glomerular disorder.

Patients in the 4C Study had more advanced CKD than patients in the ESCAPE Trial (mean eGFR, 29 vs $47 \mathrm{~mL} / \mathrm{min}$ / $\left.1.73 \mathrm{~m}^{2}\right)$. In 269 participants (30.0\%; 100 from the $4 \mathrm{C}$ Study and 169 from the ESCAPE Trial), eGFR was greater than $40 \mathrm{~mL} /$ $\mathrm{min} / 1.73 \mathrm{~m}^{2}$ at entry into their respective trial. The composite renal survival end point was reached by 402 of 898 patients (44.8\%), including 98 in the ESCAPE Trial and 304 in the 4C Study. As a consequence of the earlier progression to endstage renal disease in the more advanced patients from the $4 \mathrm{C}$ Study, the average follow-up duration was shorter in patients in the 4C Study than in the ESCAPE Trial (Table 1). All patients' characteristics stratified by CKD stage are listed in eTable 1 in the Supplement.

\section{Determinants of suPAR Concentrations}

The median (interquartile range) suPAR concentration was 5.658 (4.610-7.053) pg/mL in this study, much higher than the $2.082 \mathrm{pg} / \mathrm{mL}$ seen in healthy control participants. ${ }^{19}$ A breakdown of clinical and biochemical characteristics by suPAR distribution quartile is given in eTable 2 in the Supplement. In multivariable analysis, log suPAR concentrations were inversely associated with eGFR $(-0.009 ;$ SD, $0.001 ; P<.001)$ but were not significantly associated with sex (female, 0.042; SD, 0.022; $P=.06)$ or age $(-0.005$; SD, $0.003 ; P=.09)$. Additionally, they were not influenced by the type of renal disease.

\section{SuPAR Level and Renal Survival}

Across all patients, 5-year renal survival was 35.9\% (95\% CI, 28.7-43.0) in children with suPAR levels in the highest quartile compared with $64.5 \%$ (95\% CI, 57.4-71.7) in those in the lowest quartile $(P<.001)$ (Figure 1$)$. In multivariable analysis, older age, the presence of proteinuria, higher systolic blood pressure, glomerular and tubulointerstitial disorders, and the initial eGFR were associated with a significantly higher likelihood of reaching the renal survival end point (eTable 3 and model 1 in eTable 4 in the Supplement). The serum suPAR concentration contributed to the model (model 2 in eTable 4 in the 


\begin{tabular}{|c|c|c|c|c|c|c|}
\hline \multirow[b]{3}{*}{ Characteristic } & \multicolumn{6}{|l|}{ Mean (SD) } \\
\hline & \multicolumn{3}{|l|}{ All Patients } & \multicolumn{3}{|c|}{ Patients With eGFR $>40 \mathrm{~mL} / \mathrm{min} / 1.73 \mathrm{~m}^{2}$} \\
\hline & All & 4C Study ${ }^{14,15}$ & ESCAPE Trial ${ }^{3}$ & All & 4C Study ${ }^{14,15}$ & ESCAPE Trial $^{3}$ \\
\hline Total No. & 898 & 642 & 256 & 269 & 100 & 169 \\
\hline Age, y & $11.9(3.5)$ & $12.2(3.3)$ & $11.2(3.9)$ & $11.6(3.7)$ & $12.7(3.0)$ & $11.0(3.9)$ \\
\hline Male, No. (\%) & $560(62.4)$ & $414(64.5)$ & $146(57.0)$ & $165(61.3)$ & $72(72.0)$ & $93(55.0)$ \\
\hline \multicolumn{7}{|l|}{ Diagnosis, No. (\%) } \\
\hline CAKUT, refluxive/obstructive & $427(47.6)$ & $323(50.3)$ & $104(40.6)$ & $123(45.7)$ & $59(59.0)$ & 64 (37.9) \\
\hline CAKUT, other & $210(23.4)$ & $124(19.3)$ & $86(33.6)$ & $79(29.4)$ & $17(17.0)$ & $62(36.7)$ \\
\hline Glomerulopathies & $69(7.7)$ & $53(8.3)$ & $16(6.3)$ & $16(5.9)$ & $6(6.0)$ & $10(5.9)$ \\
\hline Tubulointerstitial disease & $92(10.2)$ & $70(10.9)$ & $22(8.6)$ & $19(7.1)$ & $8(8.0)$ & $11(6.5)$ \\
\hline Post-AKI & $42(4.7)$ & $30(4.7)$ & $12(4.7)$ & $14(5.2)$ & $4(4.0)$ & $10(5.9)$ \\
\hline Other & $58(6.5)$ & $42(6.5)$ & $16(6.3)$ & $18(6.7)$ & $6(6.0)$ & $12(7.1)$ \\
\hline Follow-up, y & $3.2(2.1)$ & $2.7(1.9)$ & $4.4(1.9)$ & $4.4(1.9)$ & $3.3(1.8)$ & $5.1(1.6)$ \\
\hline BMI & $18.3(3.9)$ & $18.5(3.9)$ & $17.9(3.7)$ & $18.2(3.7)$ & $18.5(3.7)$ & $18.1(3.8)$ \\
\hline SDS & $0.07(1.43)$ & $0.16(1.53)$ & $-0.17(1.09)$ & $-0.07(1.16)$ & $-0.05(1.29)$ & $-0.08(1.08)$ \\
\hline Height, cm & $140(21)$ & $141(20)$ & $139(22)$ & $142(22)$ & $147(19)$ & $140(23)$ \\
\hline SDS & $-1.38(1.43)$ & $-1.40(1.40)$ & $-1.31(1.50)$ & $-1.03(1.33)$ & $-1.00(1.21)$ & $-1.04(1.40)$ \\
\hline Systolic BP, mm Hg & $113(15)$ & $112(15)$ & $116(14)$ & $114(14)$ & $111(13)$ & $115.6(14.3)$ \\
\hline SDS & $0.73(1.32)$ & $0.75(1.36)$ & $0.66(1.20)$ & $0.53(1.09)$ & $0.45(1.11)$ & $0.58(1.08)$ \\
\hline Diastolic BP, $\mathrm{mm} \mathrm{Hg}$ & $69(12)$ & $69(12)$ & $70.8(12)$ & $70(11)$ & $69(10)$ & $71.5(11.2)$ \\
\hline SDS & $0.78(1.14)$ & $0.65(1.07)$ & $1.11(1.23)$ & $0.92(1.06)$ & $0.56(0.86)$ & $1.13(1.10)$ \\
\hline suPAR, $\mathrm{pg} / \mathrm{mL}$ & $6072(2247)$ & $6144(2361)$ & $5891(1925)$ & 5205 (1556) & 4638 (1139) & $5541(1671)$ \\
\hline $\mathrm{eGFR}, \mathrm{mL} / \mathrm{min} / 1.73 \mathrm{~m}^{2}$ & $34.0(15.9)$ & $28.8(11.7)$ & $47.2(17.3)$ & $54.1(11.4)$ & $48.9(10.0)$ & $57.2(11.0)$ \\
\hline Proteinuria, No. (\%) ${ }^{\mathrm{a}}$ & $664(74.5)$ & $525(82.2)$ & $139(56.3)$ & $137(51.5)$ & $61(61.0)$ & $76(45.8)$ \\
\hline Serum cholesterol level, mg/dL & $183(52)$ & $181(52)$ & $188(51)$ & $180(50)$ & $173(49)$ & $185(51)$ \\
\hline Serum albumin level, $\mathrm{g} / \mathrm{dL}$ & $4.0(0.6)$ & $3.9(0.6)$ & $4.2(0.5)$ & $4.1(0.5)$ & $4.0(0.5)$ & $4.2(0.5)$ \\
\hline Serum bicarbonate level, mEq/L & $21.7(3.70)$ & $21.2(3.8)$ & $22.7(3.0)$ & $23.1(3.0)$ & $22.7(3.2)$ & $23.3(2.9)$ \\
\hline
\end{tabular}

Abbreviations: AKI, acute kidney injury; BMI, body mass index, calculated as weight in kilograms divided by height in meters squared; BP, blood pressure; CAKUT, congenital anomalies of the kidneys and urinary tract; eGFR, estimated glomerular filtration rates; SDS, standard deviation score; suPAR, soluble urokinase receptor.

by 0.0259 ; to convert albumin level to grams per liter, multiply by 10 ; to convert bicarbonate level to millimoles per liter, multiply by 1 .

a Defined by urinary protein excretion level $\geq 0.5 \mathrm{~g} / \mathrm{g}$ creatinine or urinary albumin excretion level $\geq 50 \mathrm{mg} / \mathrm{g}$ creatinine.

SI conversion factor: To convert cholesterol level to millimoles per liter, multiply

Figure 1. Kaplan-Meier Curve of Renal Survival in 898 Children With Chronic Kidney Disease (CKD) by Soluble Urokinase Receptor (suPAR) Quartile

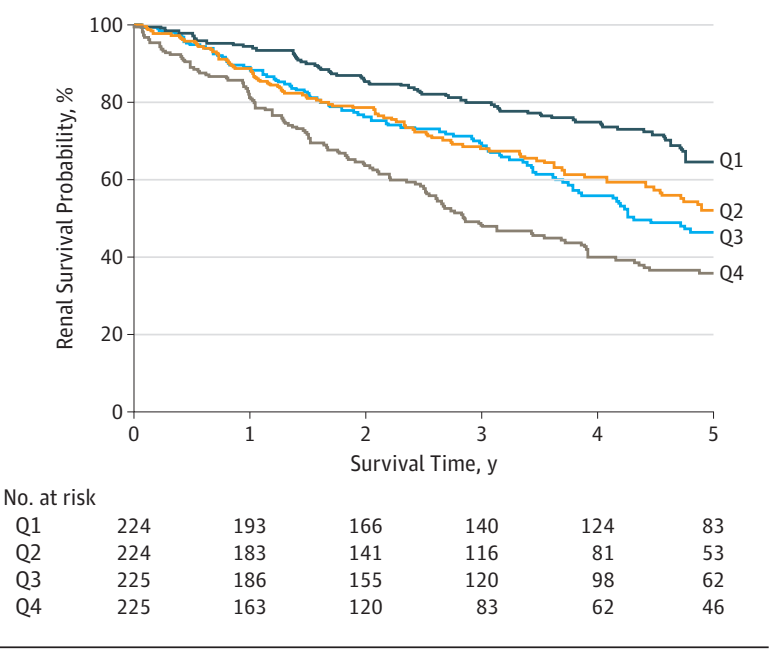

Five-year renal survival probability in children with CKD is plotted according to suPAR quartile. Q1 indicates a suPAR level less than $4.610 \mathrm{pg} / \mathrm{mL}$; Q2, 4.610-5.658 pg/mL; Q3, 5.658-7.053; Q4, greater than 7.053. Q1 is the lowest quartile and Q4 is the highest quartile.
Supplement) after adjustment for age, proteinuria, blood pressure, and renal diagnosis, but this association lost significance when initial eGFR was included in the model (model 3 in eTable 4 in the Supplement).

To determine whether the association between suPAR level and the outcome varied according to baseline eGFR, we performed sensitivity analysis using different baseline eGFR cutoff levels (30, 35, 40, 45, and $50 \mathrm{~mL} / \mathrm{min} / 1.73 \mathrm{~m}^{2}$ ). We found significant interaction between suPAR level and eGFR for the cutoff values 30,35 , and $40 \mathrm{~mL} / \mathrm{min} / 1.73 \mathrm{~m}^{2}$; the highest hazard ratio was observed for the $40 \mathrm{~mL} / \mathrm{min} / 1.73 \mathrm{~m}^{2}$ cutoff (Figure 2). None of the other covariates, including age, sex, diagnosis, or proteinuria, showed a significant interaction with respect to the association of high suPAR levels with the risk of reaching the renal survival end point (Figure 2).

Based on the observed eGFR dependence of the predictive value of suPAR levels, we performed a subgroup analysis using patients with an eGFR greater than $40 \mathrm{~mL} / \mathrm{min} / 1.73 \mathrm{~m}^{2}$. In this subgroup, proteinuria, glomerular disease, and initial eGFR were associated with a poor outcome (model 1 in Table 2 and Table 3). In addition to these factors, the circulating suPAR level was highly significantly associated with renal risk (Table 2). Accounting for both eGFR and log suPAR concentrations improved the information content of the model from an AIC of 387 
Figure 2. Forest Plot for the Progression of Renal Failure

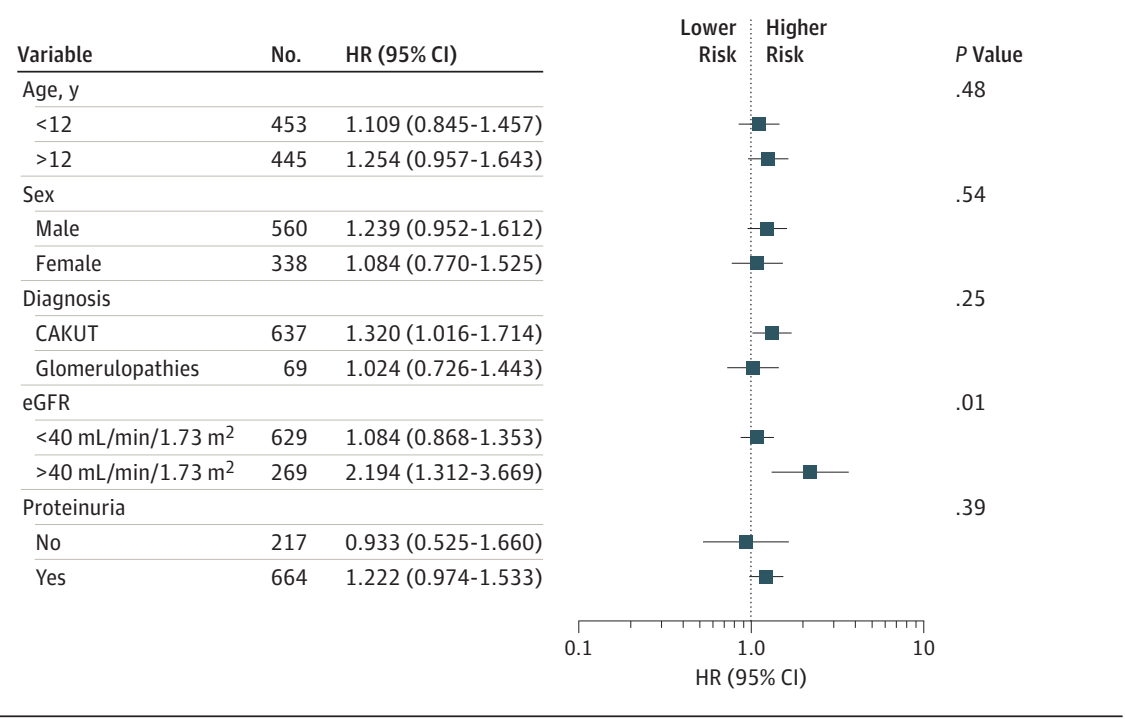

The risk attributable to high vs low soluble urokinase receptor (suPAR) levels in individual population subgroups were plotted. Each model was adjusted for all other covariates (including baseline estimated glomerular filtration rates [eGFR], age, systolic blood pressure, sex, proteinuria, and diagnosis). The $P$ value for interaction between high vs low suPAR level and the respective covariate is given. Median split for suPAR level, $5.658 \mathrm{pg} / \mathrm{mL}$. CAKUT indicates congenital anomalies of the kidneys and urinary tract; $H R$, hazard ratio. (for models including either eGFR or log suPAR) to an AIC of 381 (Table 3). Comparing the ratio of Akaike weights (0.909 vs 0.053) of model 3 with model 1 indicates that model 3 is 17.3 -fold more likely to be the best model in the AIC sense. In other words, the combined use of eGFR and suPAR level in the model was associated with a 17.3-fold lower likelihood of information loss than the use of either eGFR alone or suPAR level alone.

\section{Discussion}

This report demonstrates that serum suPAR level is associated with a more rapid decline in kidney function in pediatric patients with mild to moderate CKD at risk of progressive renal failure, independent of the established risk markers eGFR and proteinuria and across a large variety of underlying kidney diseases. To our knowledge, this is the first documentation of an association between suPAR level and loss of kidney function in children and adolescents. Given the difference between the origin of pediatric and adult kidney diseases, it was reassuring that our findings complement recent work in adult populations that associated high suPAR levels with an increased risk of developing CKD. ${ }^{11-13}$ Children with CKD represent a unique population for studies of disease progression owing to the virtual absence of confounding secondary pathologies related to factors, such as diabetes, hypertension, smoking, and aging. This allows for more sensitive assessments of biomarkers and risk factors linked to renal disease progression.

The coverage of a wide eGFR range allowed us to study the association between suPAR levels and CKD progression relative to the prevailing degree of renal dysfunction. In prospective CKD studies, GFR is universally highly predictive of renal survival because the time to both end-stage renal disease and a fractional GFR loss is shorter in patients with an initially low GFR. Owing to impaired clearance of suPAR in advanced CKD, an inverse relationship between circulating suPAR levels emerges as renal failure progresses, limiting the additional in-

\begin{tabular}{|c|c|c|c|}
\hline Parameter & $\begin{array}{l}\text { Parameter } \\
\text { Estimate }\end{array}$ & $\begin{array}{l}P \\
\text { Value } \\
\end{array}$ & $\mathrm{HR}(95 \% \mathrm{Cl})$ \\
\hline Log(suPAR) & 1.633 & .007 & $5.122(1.574-16.665)$ \\
\hline eGFR & -0.048 & .006 & $0.952(0.920-0.986)$ \\
\hline \multicolumn{4}{|l|}{ Sex } \\
\hline Female vs male & 0.282 & .39 & $1.326(0.698-2.520)$ \\
\hline Age, y & 0.081 & .09 & $1.084(0.988-1.190)$ \\
\hline \multicolumn{4}{|l|}{ Proteinuria } \\
\hline Yes & 1.021 & .003 & $2.775(1.408-5.469)$ \\
\hline \multicolumn{4}{|l|}{ Systolic BP } \\
\hline SDS & -0.019 & .88 & $0.981(0.765-1.257)$ \\
\hline \multicolumn{4}{|l|}{ Primary renal diagnosis } \\
\hline Other CAKUT & 1 [Reference] & NA & NA \\
\hline $\begin{array}{l}\text { CAKUT, } \\
\text { refluxive/obstructive }\end{array}$ & -0.735 & .07 & $0.479(0.219-1.051)$ \\
\hline Glomerulopathies & 1.618 & $<.001$ & $5.047(2.089-12.189)$ \\
\hline Post-AKI & -1.394 & .18 & $0.248(0.032-1.904)$ \\
\hline $\begin{array}{l}\text { Tubulointerstitial } \\
\text { disease }\end{array}$ & 0.643 & .24 & $1.903(0.654-5.534)$ \\
\hline Other & -0.212 & .74 & $0.809(0.230-2.850)$ \\
\hline
\end{tabular}

Abbreviations: AKI, acute kidney injury; BP, blood pressure; CAKUT, congenital anomalies of the kidneys and urinary tract; eGFR, estimated glomerular filtration rate; HR, hazard ratio; NA, not applicable; SDS, standard deviation score; suPAR, soluble urokinase receptor.

formation content of suPAR levels over eGFR alone. It remains to be explored whether the usefulness of suPAR level measurement in advanced CKD can be improved by concurrent assessment of suPAR binding and activation of its receptor on podocytes, ie, $\beta 3$-integrin..$^{10,18,20,21}$ On the other hand, suPAR has recently been demonstrated to be a sensitive predictor of early kidney damage and progression to CKD in adults. ${ }^{11-13,22}$ In this study, we provide evidence that (1) suPAR level is associated with CKD progression throughout the eGFR range from 15 to $80 \mathrm{~mL} /$ $\mathrm{min} / 1.73 \mathrm{~m}^{2}$, and (2) the strength of the association with suPAR exceeds that of eGFR alone in patients with mild to moderate 
Table 3. Effect of Estimated Glomerular Filtration Rate and Soluble Urokinase Receptor Concentrations on Renal Risk in Patients With an Estimated Glomerular Filtration Rate Greater Than $40 \mathrm{~mL} / \mathrm{min} / 1.73 \mathrm{~m}^{2}$

\begin{tabular}{|c|c|c|c|c|c|c|}
\hline \multirow[b]{3}{*}{ Parameter } & \multicolumn{2}{|l|}{ Model 1} & \multicolumn{2}{|l|}{ Model 2} & \multicolumn{2}{|l|}{ Model 3} \\
\hline & AIC $=386.6$ & & AIC $=387.2$ & & $\mathrm{AIC}=380.9$ & \\
\hline & $\mathrm{HR}(95 \% \mathrm{Cl})$ & $\begin{array}{l}P \\
\text { Value }\end{array}$ & HR $(95 \% \mathrm{Cl})$ & $\begin{array}{l}P \\
\text { Value }\end{array}$ & HR $(95 \% \mathrm{Cl})$ & $\begin{array}{l}P \\
\text { Value }\end{array}$ \\
\hline Log(suPAR) [1 unit] & NA & NA & $6.619(2.132-20.55)$ & .001 & $5.122(1.574-16.67)$ & .007 \\
\hline $\begin{array}{l}\text { eGFR, } \\
1 \mathrm{~mL} / \mathrm{min} / 1.73 \mathrm{~m}^{2}\end{array}$ & $0.945(0.914-0.978)$ & .001 & NA & NA & $0.952(0.920-0.986)$ & .006 \\
\hline Female sex & $1.135(0.607-2.123)$ & .69 & $1.254(0.663-2.370)$ & .49 & $1.326(0.698-2.520)$ & .39 \\
\hline Age, 1 y & $1.054(0.967-1.150)$ & 23 & $1.061(0.970-1.160)$ & .20 & $1.084(0.988-1.190)$ & .09 \\
\hline $\begin{array}{l}\text { Proteinuria group, } \\
\text { yes }\end{array}$ & $3.141(1.587-6.216)$ & .001 & $2.984(1.519-5.861)$ & .002 & $2.775(1.408-5.469)$ & .003 \\
\hline Systolic BP, 1 SDS & $0.987(0.764-1.274)$ & .92 & $0.922(0.720-1.180)$ & .52 & $0.981(0.765-1.257)$ & .88 \\
\hline $\begin{array}{l}\text { CAKUT, } \\
\text { refluxive/obstructive }\end{array}$ & $0.584(0.274-1.246)$ & .16 & $0.480(0.020-1.050)$ & .07 & $0.479(0.219-1.051)$ & .07 \\
\hline Glomerulopathy & $5.702(2.333-13.93)$ & $<.001$ & $4.321(1.812-10.30)$ & .001 & $5.047(2.089-12.19)$ & $<.001$ \\
\hline Post-AKI & $0.252(0.033-1.933)$ & .19 & $0.268(0.035-2.060)$ & .21 & $0.248(0.032-1.904)$ & .18 \\
\hline Tubulointerstitial & $2.276(0.789-6.561)$ & .13 & $1.684(0.579-4.899)$ & .34 & $1.903(0.654-5.534)$ & .24 \\
\hline Other & $0.863(0.246-3.032)$ & .82 & $0.729(0.209-2.546)$ & .001 & $0.809(0.230-2.850)$ & .74 \\
\hline
\end{tabular}

Abbreviations: AIC, Akaike information criterion; AKI, acute kidney injury; BP, blood pressure; CAKUT, congenital anomalies of the kidneys and urinary tract; eGFR, estimated glomerular filtration rate; HR, hazard ratio; NA, not applicable; SDS, standard deviation score; suPAR, soluble urokinase receptor.
CKD, ie, eGFR ranging from 40 to $80 \mathrm{~mL} / \mathrm{min} / 1.73 \mathrm{~m}^{2}$. The association between suPAR level and the rate of decline in kidney function was independent of the presence of significant proteinuria, the other well-established risk marker of CKD progression. Hence, suPAR level has the potential to become a clinically useful marker for risk stratification in patients with mild to moderate CKD (stage 3), the critical phase when secondary complications of CKD start to develop and nephroprotective interventions may be most effective.

Another important observation was the independence of the association of suPAR concentrations with CKD progression of the underlying kidney disease. Chronic kidney disease in children is predominantly caused by congenital renal malformations and hereditary kidney disorders, in striking contrast to the adult population, where CKD in most individuals is secondary to diabetes, hypertension, immunoglobulin A nephropathy, or aging. Whereas adult CKD usually originates from glomerular pathology, more than $70 \%$ of the pediatric CKD cohort studied here originated from congenitally reduced functioning nephron mass, with another $10 \%$ from primary tubulointerstitial disorders, and only $7 \%$ of our study population had primary glomerular disease. Despite the differences in etiology, children and adults with CKD exhibit similar rates of progression.

Soluble urokinase receptor belongs to the lysine-6/uPAR/ a-neurotoxin family of signaling proteins and was originally identified as a circulating factor in glomerular disorders. ${ }^{10}$ Our findings that eGFR-adjusted suPAR levels were similar across the disease groups and that suPAR level was associated with the trajectory of kidney function over time regardless of the underlying disease imply that its predictive value is not limited to glomerular disease. Soluble urokinase receptor is known to be produced by bone marrow-derived immature myeloid cells, and in preclinical studies, it contributes to systemic forms of kidney disease commonly associated with proteinuria. ${ }^{23}$ In animal models, animals with elevated suPAR levels develop renal disease, ${ }^{10,23,24}$ and one can speculate about potential sites of involvement of suPAR along the nephron. For example, suPAR binds to and activates $\beta 3$-integrins and triggers alterations in podocyte structure and function. ${ }^{10,25,26}$ Whether suPAR acts in the tubulointerstitium to promote fibrosis in individuals with congenital anomalies or primary tubulointerstitial diseases requires further study.

The mean suPAR level in our cohorts was often several-fold higher than levels measured in children with normal weight or obesity, ${ }^{27}$ and it was comparable with or higher than in adults who will develop kidney disease. Also, suPAR levels tended to decrease with patient age. We speculate that a more active innate immune system in childhood may lead to higher suPAR levels. ${ }^{11,12}$

\section{Strengths and Limitations}

The strengths of this study are the large numbers of pediatric patients enrolled in 2 high-quality clinical studies. The population encompasses a wide range of underlying renal diseases, age, and eGFR and represents a diversity of racial/ethnic backgrounds, macroeconomic factors, and health care systems across 13 European countries. Patients were comprehensively phenotyped over time so that the longitudinal data on GFR are likely to be accurate. The composite renal survival end point in this study represents a hard clinical outcome consistent with the standard that the US Food and Drug Administration applies to assess the efficacy of novel therapeutic interventions.

The limitations of this study include measurement of suPAR levels at a single time, the lack of African American and Asian individuals, and the lack of complete clinical information to eliminate residual confounding factors (eg, smoking, environmental exposures, and birth weight). Finally, because our patient population consisted of patients with established CKD, we are unable to derive conclusions on the usefulness of suPAR level as a predictive marker in patients at risk for or in very early stages of CKD.

\section{Conclusions}

In conclusion, our findings demonstrate that an elevated serum suPAR level is associated with increased risk of deterioration in kidney function in children with mild to moderate CKD (eGFR, $40-80 \mathrm{~mL} / \mathrm{min} / 1.73 \mathrm{~m}^{2}$ ) secondary to a broad range of 
renal disorders. Given that suPAR level predicts CKD development in various at-risk populations, ${ }^{13}$ it is plausible that measurement of serum suPAR level early in the course of pediatric CKD may identify patients at heightened risk of progressive kidney function loss and adverse clinical outcomes. This is es- pecially relevant in pediatric nephrology practice because at least half of all CKD cases are caused by CAKUT or hereditary disorders that can be identified during infancy or even prenatally. Ongoing mechanistic studies have the potential to define suPAR level as a prime target for renal therapeutics.

\section{ARTICLE INFORMATION}

Accepted for Publication: July 6, 2017.

Correction: This article was corrected on October 9 ,

2017, to fix an error in the presentation of data

in Table 1.

Published Online: September 5, 2017.

doi:10.1001/jamapediatrics.2017.2914

Author Affiliations: Center for Pediatrics and

Adolescent Medicine, University Hospital Heidelberg, Heidelberg, Germany (Schaefer, Wühl); Department of Pediatrics, Division of Nephrology, New York University Langone Medical Center, New York (Trachtman); Institute of Medical Biometry and Informatics, University of Heidelberg, Heidelberg, Germany (Kirchner); Emory Clinical Cardiovascular Research Institute, Division of Cardiology, Emory University School of Medicine, Atlanta, Georgia (Hayek); Department of Pediatric Nephrology, Faculty of Medicine, Cukurova University, Adana, Turkey (Anarat); Pediatric Nephrology, Hacettepe University Faculty of Medicine, Ankara, Turkey (Duzova); Pediatric Nephrology, Ege University Faculty of Medicine, Izmir, Turkey (Mir); University Children's Hospital Belgrade, Belgrade, Serbia (Paripovic); Department of Pediatric Nephrology, Istanbul Medical Faculty, Istanbul, Turkey (Yilmaz); Pediatric Nephrology, Giannina Gasline Institute, Genova, Italy (Lugani); Pediatric Nephrology, Vienna University Children's Hospital, Vienna, Austria (Arbeiter); Nephrology, Kidney Transplantation and Hypertension, Children's Memorial Health Institute, Warzaw, Poland (Litwin); Pediatric Nephrology, Children's Hospital, University Medical Center Hamburg-Eppendorf, Hamburg, Germany (Oh); Division of Pediatric Nephrology, Bambino Gesù Children's Hospital and Research Institute, Rome, Italy (Matteucci); Pediatric Nephrology, Charité Children's Hospital, Berlin, Germany (Gellermann); Children's Dialysis Center, Hospital St Georg, Leipzig, Germany (Wygoda); Vilnius University, Pediatric Center, Vilnius, Lithuania (Jankauskiene); KfH Kidney Center for Children, Marburg, Germany (Klaus); Pediatrics, University Hospital Motol, Prague, Czech Republic (Dusek); Pediatric Nephrology and Dialysis, Fondazione OSP Maggiore Policlinico, Milano, Italy (Testa); Department of Pediatric and Adolescent Nephrology, Medical University Gdańsk, Gdańsk, Poland (Zurowska); Pediatrics, Hospital São João, Porto, Portugal (Caldas Afonso); Department of Medicine, Rush University Medical Center, Chicago, Illinois (Tracy, Wei, Reiser); Harvard Medical School and Division of Nephrology, Massachusetts General Hospital, Charlestown (Sever); The Research Institute at Nationwide Children's Hospital, The Ohio State University, Columbus (Smoyer).

Author Contributions: Drs Schaefer and Kirchner had full access to all of the data in the study and take responsibility for the integrity of the data and the accuracy of the data analysis. Drs Schaefer, Trachtman, Wühl, and Reiser contributed equally to the preparation of this article.

Study concept and design: Schaefer, Trachtman, Wühl, Hayek, Anarat, Yilmaz, Lugani, Caldas Afonso, Sever, Smoyer.

Acquisition, analysis, or interpretation of data:
Schaefer, Trachtman, Wühl, Kirchner, Hayek, Duzova, Mir, Paripovic, Lugani, Arbeiter, Litwin, Oh, Matteucci, Gellermann, Jankauskiene, Klaus, Dusek, Testa, Zurowska, Tracy, Wei, Reiser.

Drafting of the manuscript: Schaefer, Trachtman, Wühl, Kirchner, Hayek, Anarat, Yilmaz, Matteucci, Dusek,

Zurowska, Tracy, Wei.

Critical revision of the manuscript for important

intellectual content: Trachtman, Wühl, Kirchner, Hayek, Duzova, Mir, Paripovic, Yilmaz, Lugani, Arbeiter, Litwin, Oh, Gellermann, Jankauskiene, Klaus, Testa, Caldas Afonso, Wei, Sever, Smoyer, Reiser. Statistical analysis: Wühl, Kirchner, Hayek, Matteucci. Obtained funding: Schaefer, Mir, Gellermann. Administrative, technical, or material support: Schaefer, Duzova, Paripovic, Yilmaz, Lugani, Arbeiter, Oh, Klaus, Dusek, Zurowska, Caldas Afonso, Wei, Smoyer, Reiser.

Supervision: Schaefer, Lugani, Sever.

Conflict of Interest Disclosures: Drs Sever and Reiser are inventors on pending and issued patents related to antiproteinuric therapies. They stand to gain royalties from present and future commercialization. They also are cofounders and advisors to TRISAQ, a biotechnology company. Dr Wei has a pending patent on suPAR in diabetes. He stands to gain royalties from future commercialization products concerning this application. No other conflicts were disclosed. Funding/Support: Dr Schaefer received support for this study from grant agreement 2012-305608 (EURenOmics) from the European Community Seventh Framework Programme (FP7/2007-2013). The ESCAPE Trial was supported by grants from the Boehringer Ingelheim Stiftung, the European Commission Fifth Framework Programme (grant QLRT-2001-00908), the Kuratorium für Dialyse und Nierentransplantation Neu-Isenburg $(\mathrm{KfH})$, and the Baxter Extramural Grant Program. Support for the 4C Study was received from the European Renal Association-European Dialysis and Transplant Association Research Programme, the $\mathrm{KfH}$ Foundation for Preventive Medicine, and the German Federal Ministry of Education and Research (grant 01EO0802). Drs Wei, Sever, and Reiser were supported by grant R01DK101350 from the National Institute of Diabetes and Digestive and Kidney Diseases. Dr Trachtman was supported by grant R01DK100307 from the National Institute of Diabetes and Digestive and Kidney Diseases. Role of the Funder/Sponsor: The funders had no role in the design and conduct of the study; collection, management, analysis, and interpretation of the data; preparation, review, or approval of the manuscript; and decision to submit the manuscript for publication.

Group Information: The principal investigators of the ESCAPE Trial included the following: Cukurova University School of Medicine, Adana, Turkey: Ali Anarat, MD; Hacettepe University Faculty of Medicine, Ankara, Turkey: Aysin Bakkaloglu, MD, and Fatih Ozaltin, MD; University Children's Hospital, Belgrade, Serbia: Amira Peco-Antic, MD; Charité Children's Hospital, Berlin, Germany: Uwe Querfeld, MD, and Jutta Gellermann, MD; First Department of
Pediatrics, Semmelweis University Budapest, Budapest, Hungary: Peter Sallay, MD; PolishAmerican Children's Hospital, Jagiellonian University Collegium Medicum, Krakow, Poland: Dorota Drożdż, MD; University Hospital Essen, Essen, Germany Klaus-Eugen Bonzel, MD, and Anna-Margrete Wingen, MD; Department of Paediatric and Adolescent Nephrology and Hypertension, Medical University of Gdańsk, Gdańsk, Poland: Aleksandra Żurowska, MD, and Irena Balasz, MD; Giannina Gasline Institute, Genova, Italy: Antonella Trivelli, MD, and Francesco Perfumo, MD; University Children's Hospital Hamburg-Eppendorf, Hamburg, Germany: Dirk-Erhard Müller-Wiefel, MD, and Kerstin Möller, MD; Hannover Medical School, Children's Hospital, Hannover, Germany: Gisela Offner, MD, and Barbara Enke, MD; Center for Pediatric and Adolescent Medicine, Heidelberg, Germany: Elke Wühl, MD, Charlotte Hadtstein, MD, Otto Mehls, MD, and Franz Schaefer, MD; Istanbul Medical Faculty, Istanbul University, Çapa campus, Istanbul, Turkey: Sevinc Emre, MD; Cerrahpaşa Faculty of Medicine, Istanbul University, Istanbul, Turkey: Salim Caliskan, MD; Department of Pediatrics, Ege University Faculty of Medicine, İzmir, Turkey: Sevgi Mir, MD; City Hospital St Georg, Leipzig, Germany: Simone Wygoda, MD; University Children's Hospital, Mainz, Germany: Katharina Hohbach-Hohenfellner, MD; Department of Pediatrics, Philipps University Marburg, Marburg, Germany: Nickola Jeck, MD, and Günter Klaus, MD; IRCCS Ospedale Maggiore, Policlinico-Mangiagalli, Milano, Italy: Gianluigi Ardissino, MD, and Sara Testa, MD; Azienda Ospedaliera, Università di Padova, Padova, Italy: Giovanni Montini, MD; Hôpital Necker, Paris, France: Marina Charbit, MD, and Patrick Niaudet, MD; Hospital de São João, Porto, Portugal: Alberto Caldas Afonso, MD, and Ana FernandesTeixeira, MD; Department of Pediatrics, University Hospital Motol, Prague, Czech Republic: Jiri Dušek, MD; Ospedale Pediatrico Bambino Gesù, Rome, Italy: Chiara Matteucci, MD, and Stefano Picca, MD; University Children's Hospital, Rostock, Germany: Marianne Wigger, MD; Karolinska Institute, Huddinge University Hospital, Stockholm, Sweden: Ulla B. Berg, MD, and Giovanni Celsi, MD; Pediatrie 1, Hopitaux Universitaires de Strasbourg, Strasbourg, France: Michel Fischbach, MD, and Joelle Terzic, MD; Pomeranian Academy of Medicine, Szczecin, Poland: Janusz Fydryk, MD, and Tomasz Urasinski, MD; Ospedale Infantile Regina Margherita, Torino, Italy: Rosanna Coppo, MD, and Licia Peruzzi, MD; University Children's Hospital, Vienna, Austria: Klaus Arbeiter, MD; Vilnius University Children's Hospital, Vilnius University, Vilnius, Lithuania: Augustina Jankauskiené, MD; Children's Memorial Health Hospital, Warsaw, Poland: Ryszard Grenda, MD, and Mieczyslaw Litwin, MD; and University Children's Hospital, Zürich, Switzerland: Thomas J. Neuhaus, MD. The principal investigators of the 4C Study included the following: Pediatric Nephrology, University Children's Hospital Vienna, Vienna, Austria: Klaus Arbeiter, MD; Department of Pediatrics I, Medical University, Innsbruck, Austria: Alejandra Rosales, MD; University Hospital Motol, Prague, Czech Republic: Jiri Dusek, MD; Pole Medico- 
Chirugical de Pediatrie, Hôpital de Hautepierre Strasbourg France: Ariane Zaloszyc, MD; Pediatric Nephrology, Charité Campus Virchow-Klinikum, Berlin, Germany: Uwe Querfeld, MD, and Jutta Gellermann, MD; Pediatric Nephrology Immunology and Hypertensiology, University Children's Hospital, Cologne, Germany: Max Liebau, MD, and Lutz Weber, MD; Pediatric Nephrology, University Children's Hospital, Erlangen, Germany: Evelin Muschiol, MD; Pediatric Nephrology, University Children's Hospital, Essen, Germany: Rainer Büscher, MD; Pediatric Nephrology, UKE University Children's Hospital, Hamburg, Germany: Jun Oh, MD; Pediatric Nephrology, Hannover Medical School, Hannover, Germany: Anette Melk, MD, Daniela Thurn-Valassina, MD, and Dieter Haffner, MD; Division of Pediatric Nephrology, Center for Children and Adolescents, Heidelberg, Germany: Franz Schaefer, MD; Division of Pediatric Nephrology, Center for Pediatrics and Adolescent Medicine, Freiburg, Germany: Charlotte Gimpel, MD; Division of Pediatric and Nephrology, Center for Pediatrics and Adolescent, Jena, Germany: Ulrike John, MD; Children's Dialysis Center, Town Hospital St Georg, Leipzig, Germany: Simone Wygoda MD; KfH Kidney Center for Children, Marburg, Germany: Nikola Jeck, MD; Pediatric Nephrology, University Children's Hospital, Rostock, Germany: Marianne Wigger, MD; Pediatric Nephrology and Dialysis, Ospedale Maggiore, Policlinico, Milano, Italy: Sara Testa Fondazione, MD; Dialysis and Transplantation Department of Pediatrics, Dipartimento di Pediatria Salus Pueri, Padova, Italy: Luisa Murer, MD; Division di Nefrologia e Dialisi, Ospedale Pediatrico Bambino Gesú, Roma, Italy: Chiara Matteucci, MD; Center for Pediatrics, University Children's Hospital, Vilnius, Lithuania: Augustina Jankauskiene, MD, and Karolis Azukaitis, MD; Dialysis Unit, PA Children's Hospital, Cracow, Poland: Dorota Drozdz, MD; Pediatric Nephrology, Instituto Giannina Gaslini, Genova, Italy: Francesca Lugani, MD; Department of Pediatric and Adolescent Nephrology, University Medical School, Gdańsk, Poland: Aleksandra Zurowska, MD; Pomeranian Academy of Medicine, Clinic of Pediatrics, Szczecin, Poland: Marcin Zaniew, MD; Nephrology, Kidney, Transplantation and Hypertension, Children's Memorial Health Institute, Warsaw, Poland: Mieczyslaw Litwin, MD, and Anna Nimierska, MD; Serviçio de Pediatria, Hospital de São João, Porto, Portugal: Ana Teixeira, MD; University Children's Hospital, Belgrade, Serbia: Amira Peco-Antic, MD, and Dusan Paripovic, MD; Nephrology, University Children's Hospital, Zürich, Switzerland: Guido Laube, MD; Cocuk Nefrolojisi Bilim Dali, Cukurova Universitesi Tip Fakültesi, Adana, Turkey: Ali Anarat, MD, and Aysun Bayazit, MD; Pediatric Nephrology, Hacettepe University, Ankara, Turkey: Ali Duzova, MD, and Yelda Bilginer, MD; Pediatric Nephrology, Cerrahpasa Medical Faculty, Istanbul, Turkey: Salim Caliskan, MD, Nur Canpolat, MD, and Mahmut Civilibal, MD; Pediatric Nephrology, Ege University، Izmir, Turkey: Sevgi Mir, MD, and Betül Sözeri, MD; Pediatric Nephrology, University Children's Hospital, Münster, Germany: Brigitta Kranz, MD; Department of Pediatrics, Sant'Orsola-Malphighi Hospital, Bologna, Italy: Francesca Mencarelli, MD; Inselspital Children's Hospital, Bern, Switzerland: Brigitte Dorn, MD; Pediatric Nephrology, University Tip Faculty of Medicine Cebeci Kampusu Cocuk, Ankara, Turkey: Fatos Yalcinkaya, MD; Faculty of Medicine, Baskent University, Ankara, Turkey: Esra Baskin, MD; Diskapi Children's Hospital, Ankara, Turkey: Nilgun Cakar, MD; Pediatric Nephrology, Gazi University Hospital,
Ankara, Turkey: Oguz Soylemezoglu, MD; Department of Pediatric Nephrology, Istanbul Medical Faculty, Çapa-Istanbul, Turkey: Sevinc Emre, MD; Pediatric Nephrology, Goztepe Educational and Research Hospital, Istanbul, Turkey: Cengiz Candan, MD; Pediatric Nephrology, Bakirkoy Children's Hospital, Istanbul, Turkey: Aysel Kiyak, MD; Pediatric Nephrology, Sisli Educational and Research Hospital, Istanbul, Turkey: Gul Ozcelik, MD; Pediatric Nephrology, Marmara University Medical Faculty, Istanbul, Turkey: Harika Alpay, MD; Nephrology, Great Ormond Street Hospital, London, United Kingdom: Rukshana Shroff, MD; Service de Néphrologie Pédiatrique, Universite Hôpital Femme Mère Enfant, Lyon, France: Bruno Rachin, MD; Service de Pédiatrie, Centre de Reference Maladies Renales Rares du Sud-Quest, Bordeaux, France: Jerome Harambat, MD; Pediatric Nephrology, Zabrze, Poland: Maria Szczepanska, MD; Dortcelik Children's Hospital Bursa, Turkey: Hakan Erdogan, MD; Uludag University, Bursa, Turkey: Osman Donmez, MD; Department of Pediatric Nephrology, University of Gaziantep, Gaziantep, Turkey: Ayse Balat, MD; Tepecik Training and Research Hospital, Izmir, Turkey: Nejat Aksu, MD; Department of Pediatric Nephrology, Inonu Universtiy Medical School, Malatya, Turkey: Yilmaz Tabel, MD; Pediatric Nephrology Department, Celal Bayar University, Manisa, Turkey: Pelin Ertan, MD; and Pediatric Nephrology, Sanliurfa Children's Hospital, Sanliurfa, Turkey: Ebru Yilmaz, MD.

\section{REFERENCES}

1. Wong CJ, Moxey-Mims M, Jerry-Fluker J, Warady BA, Furth SL. CKiD (CKD in children) prospective cohort study: a review of current findings. Am J Kidney Dis. 2012;60(6):1002-1011.

2. Warady BA, Chadha V. Chronic kidney disease in children: the global perspective. Pediatr Nephrol. 2007;22(12):1999-2009.

3. Wühl E, Trivelli A, Picca S, et al; ESCAPE Trial Group. Strict blood-pressure control and progression of renal failure in children. $N$ Engl J Med. 2009;361(17):1639-1650.

4. Rodig NM, McDermott KC, Schneider MF, et al. Growth in children with chronic kidney disease: a report from the Chronic Kidney Disease in Children Study. Pediatr Nephrol. 2014;29(10):1987-1995. 5. Shroff R, Aitkenhead H, Costa N, et al; ESCAPE Trial Group. Normal 25-hydroxyvitamin D levels are associated with less proteinuria and attenuate renal failure progression in children with CKD. J Am Soc Nephrol. 2016;27(1):314-322.

6. Ingelfinger JR, Kalantar-Zadeh K, Schaefer F; World Kidney Day Steering Committee. Averting the legacy of kidney disease: focus on childhood. J Nephrol. 2016;29(2):137-142.

7. Luck M, Bertho G, Bateson M, et al. Rule-mining for the early prediction of chronic kidney disease based on metabolomics and multi-source data. PLoS One. 2016;11(11):e0166905.

8. Hsu CY, Xie D, Waikar SS, et al; CRIC Study Investigators; CKD Biomarkers Consortium. Urine biomarkers of tubular injury do not improve on the clinical model predicting chronic kidney disease progression. Kidney Int. 2017;91(1):196-203. 9. Alderson HV, Ritchie JP, Pagano S, et al. The associations of blood kidney injury molecule-1 and neutrophil gelatinase-associated lipocalin with progression from CKD to ESRD. Clin J Am Soc Nephrol. 2016;11(12):2141-2149.

10. Wei C, El Hindi S, Li J, et al. Circulating urokinase receptor as a cause of focal segmental glomerulosclerosis. Nat Med. 2011;17(8):952-960.
11. Hayek SS, Sever S, Ko YA, et al. Soluble urokinase receptor and chronic kidney disease. N Engl J Med. 2015;373(20):1916-1925.

12. Park MY, Herrmann SM, Saad A, et al. Biomarkers of kidney injury and klotho in patients with atherosclerotic renovascular disease. Clin J Am Soc Nephrol. 2015;10(3): 443-451.

13. Schulz CA, Persson M, Christensson A, et al. Soluble urokinase-type plasminogen activator receptor (suPAR) and impaired kidney function in the population-based Malmö Diet and Cancer Study. Kidney Int Rep. 2017;2(2):239-247. 14. Querfeld U, Anarat A, Bayazit AK, et al; $4 C$ Study Group. The Cardiovascular Comorbidity in Children with Chronic Kidney Disease (4C) study: objectives, design, and methodology. Clin J Am Soc Nephrol. 2010;5(9):1642-1648.

15. Schaefer F, Doyon A, Azukaitis K, et al; 4C Study Consortium. Cardiovascular phenotypes in children with CKD: The 4C Study. Clin J Am Soc Nephrol. 2017;12(1):19-28.

16. von Elm E, Altman DG, Egger M, Pocock SJ, Gøtzsche PC, Vandenbroucke JP; STROBE Initiative. The Strengthening the Reporting of Observational Studies in Epidemiology (STROBE) statement: guidelines for reporting observational studies. Ann Intern Med. 2007;147(8):573-577.

17. WagenmakersEJ, FarrellS. AIC model selection using Akaike weights. Psychon Bull Rev. 2004;11(1):192-196. 18. Li F, Zheng C, Zhong Y, et al. Relationship between serum soluble urokinase plasminogen activator receptor level and steroid responsiveness in FSGS. Clin J Am Soc Nephrol. 2014;9(11):1903-1911. 19. Wei C, Trachtman H, Li J, etal; PodoNet andFSGSCT Study Consortia. Circulating suPAR in two cohorts of primary FSGS. J Am Soc Nephrol. 2012;23(12): 2051-2059.

20. Spinale JM, Mariani LH, Kapoor S, et al; Nephrotic Syndrome Study Network. A reassessment of soluble urokinase-type plasminogen activator receptor in glomerular disease. Kidney Int. 2015;87(3):564-574. 21. Sinha A, Bajpai J, Saini S, et al. Serum-soluble urokinase receptor levels do not distinguish focal segmental glomerulosclerosis from other causes of nephrotic syndrome in children. Kidney Int. 2014;85 (3):649-658

22. Guthoff $M$, Wagner $R$, Randrianarisoa $E$, et al. Soluble urokinase receptor (suPAR) predicts microalbuminuria in patients at risk for type 2 diabetes mellitus. Sci Rep. 2017;7:40627.

23. Hahm E, Wei C, Fernandez I, et al. Bone marrowderived immature myeloid cells are a main source of circulating suPAR contributing to proteinuric kidney disease. Nat Med. 2017;23(1):100-106.

24. Alfano $M$, Cinque $P$, Giusti $G$, et al. Full-length soluble urokinase plasminogen activator receptor down-modulates nephrin expression in podocytes. Sci Rep. 2015;5:13647.

25. Yoo TH, Pedigo CE, Guzman J, et al. Sphingomyelinase-like phosphodiesterase 3b expression levels determine podocyte injury phenotypes in glomerular disease. J Am Soc Nephrol. 2015;26(1):133-147.

26. Hayek SS, Koh KH, Grams ME, et al. A tripartite complex of suPAR, APOL1 risk variants and av $\beta 3$ integrin on podocytes mediates chronic kidney disease [published online June 26, 2017]. Nat Med. 27. Kosecik M, Dervisoglu P, Koroglu M, etal. Usefulness of soluble urokinase plasminogen activator receptor (suPAR) as an inflammatory biomarker in obese children. Int J Cardiol. 2017;228:158-161. 